\title{
YOUR BRAIN ON NARRATION
}

\author{
Stefano F. Cappa
}

\section{Institute for Advanced Studies (IUSS-Pavia); IRCCS S. Giovanni di Dio Fatebenefratelli Brescia, Italy}

\begin{abstract}
The study of the brain mechanisms involved in narrative production and comprehension has made significant progress in the last decade. The paper reviews some of the available evidence, focusing on functional imaging studies aimed to assess brain activity in normal subjects, while they listen to narratives or watching movies. The complex brain networks engaged by these complex tasks extend beyond the classical language areas, by involving the right hemisphere and hippocampal structures. These results provide initial insights into the mechanisms involved in narrative cohesion, situation model construction and distinction/integration of narrative elements.
\end{abstract}

Key words: narration, functional brain imaging, hippocampus.

\section{INTRODUCTION}

Narrative abilities can be investigated at multiple levels, and the contribution of neuroscience to the understanding of the brain mechanisms of narration is just starting to be appreciated.

It must be acknowledged that some crucial aspects, such as artistic creativity, remain hardly accessible to empirical investigation (see some examples of the approaches used in this area of research in $[1,2])$. In spite of the complex nature of the topic, significant insights can be gained from the careful investigation of artists affected by neurological disorders. An excellent examples is the study of Iris Murdoch's last writings by Garrard and colleagues [3]. On the other hand, brain mechanisms involved in generic narrative production and comprehension are accessible to direct investigation, making it possible to achieve significant progress in the last decade.

The cognitive neuroscience of language has been for many years largely based on an analytic approach, aiming to understanding the brain mechanisms involved in tasks such as phonological discrimination of syllables or retrieval of single lexical item [4]. While these tasks, which have been extensively applied in studies of aphasic patients as well as in functional imaging, are clearly very distant from natural language use, they have been extremely useful for understanding the neurological foundations of speech perception and production and of lexical semantic processing. The study of syntactic processing is clearly required to extend the investigation to the sentence level, but this was largely done using metalinguistic tasks, such as detection of syntactic violations and sentence-picture matching [5]. Discourse and textual level analysis has a long-standing tradition in psycholinguistics. According to a classic model of discourse comprehension [6] a reader understanding of a text requires the construction of three types of representation. The surface representation is the mental representation of the words and structure of the text. The text base is the representation of the propositions included in the text, independent of their form. The situation model is the representation of a situation and of a set of events. A core property at the surface level is cohesion among the linguistic elements, different from coherence, which refers to connections among elements taking place at the text base or situation level. Model construction during narration is a dynamic process, requiring continuous changes to specific situation models (mapping), as well as shifting to different levels [7].

These models have had a limited impact on language neuroscience research, and mostly on the investigation of the consequences of brain damage. There is a considerable literature on the effect of neurological and psychiatric disorders on discourse and narrative processing. Disorders of intersentential cohesion, for example, have been considered as a typical feature of narrative production in traumatic brain injury patients [8]. Macrolinguistic abilities are also typically impaired in patients with schizophrenia [9]. These studies are of great theoretical and clinical interest, but their contribution to the understanding of the brain mechanisms supporting narrative production and compre- 
hension is limited. More advanced insights have been provided by investigations using neuroimaging methods, which allow to explore brain activity in normal subjects engaged in cognitive tasks.

\section{NARRATION IN TEXTS}

Narrative processing has been investigated in a handful of functional magnetic resonance (fMRI) studies. An early study [10] assessed brain activity while subjects were listening to Aesop's fables, presented as random words, individual, unconnected sentences, or as coherent narratives. The main result was that listening to coherent narratives elicited brain activation extending beyond the classical language areas of the dominant hemisphere, recruiting precuneus, medial prefrontal, and dorsal temporo-parieto-occipital cortices. The non-dominant (right hemisphere) was also engaged by the coherent stories, at the time of resolution of a story. Another study [11] specifically addressed the brain correlates of coherence. Related vs unrelated sentences activated an extensive bilateral cortical network, which may reflect the retrieval and integration of information form semantic memory required for the inferencing process. An interesting manipulation of coherence was performed in [12]. The subjects listened to stories, which were overall coherent, but difficult to understand if a title was not provided. The pattern of brain activity was more extensive, in the left but also in the right hemisphere, when the title was provided. The authors interpret this finding as reflecting the construction of a more complex situational model in the case of the processing of the globally coherent narration provided by the title. Recently, a few studies have applied more sophisticated data analysis approaches than the classical subtraction design used by the previous investigations. For example, functional connectivity analysis [13] was applied to fMR data of subjects listening to narratives, which were preceded by a congruent or incongruent picture. The main finding was that in the case of congruent pictures Broca's area, a crucial language region, increased its connectivity with frontal and parietal regions, in particular with the angular gyrus. A remarkable recent study [14] applied the predictive coding model [15] to the analysis of brain activity during narrative discourse comprehension. The general model posits that language processing depends upon a hierarchically organized predictive network, based on nested internal models generating temporal predictions for incoming information at all levels (sounds, words, sentences, discourse). Listening to narratives, discourse-related cues, such as a passive voice, results in an increased predictability of the presence of a character later in the story. This kind of manipulation yielded a decrease in brain activity within the "dorsal auditory stream" con- necting the inferior parietal lobule with frontal areas. Finally, an impressive series of studies from Gallant's lab applies one of the more advanced data-driven approaches to the investigation of the brain correlates of linguistic processing in subjects listening to naturally spoken narrative stories [16]. The data reported have up to now focused on the domain-specific mapping of the semantic space in the human cortex [17], but the methodology can be prospectively applied to other aspects of language processing, including discourse level analysis.

To summarize, brain activity is more extensive when subjects listen to narratives, rather than to words or isolated sentences. Multiple brain areas, beyond the classical language regions, are involved, extending to the non-dominant hemisphere. This increase in the extent of brain activity is modulated by coherence and by the construction of situation models. Within the perisylvian language areas, activity modulation and connectivity changes may reflect the modifications of processing load associated with discourse analysis.

\section{NARRATION IN MOVIES}

The investigation of brain activity in subjects watching movies has a relatively long story, and has played a crucial role in methodological developments, such as multivariate data-driven analysis. A seminal example is the study of intersubject correlation of brain activity during free viewing of the movie by S. Leone, The Good the Bad and the Ugly [18]. The presence of a high level of spatio-temporal synchronization allowed to assess the "reverse correlation" of specific events, such as emotional scenes, to patterns of brain activity. A development of this approach [19] is at the basis of the Gallant's lab method mentioned above [16]. Also in the case of movies most of the studies deal with the brain correlates of semantic representation, but a 20 remarkable exception is a recent paper focused on the narrative aspect of movies [21]. The subjects were scanned while watching Sliding Doors, a movie by P. Howitt, which is characterized by two interleaving narratives diverging from a common beginning. This allowed to assess the brain correlates of the emergence of the two competing narratives and provided important insight on the central role played by the hippocampus, the core structure for episodic memory formation, during the unfolding of a narrative. In the first place, the hippocampus was involved in the differentiation of person and place, as indicated by differential response to character and location. The second distinction at the hippocampal level was between the two different stories, which remarkably increased over time with the divergence of the narratives. The coordination of these two aspects, distinction of elements and integration of contexts, may 
be a common feature of the hippocampal contribution to other functions, such as spatial representation and autobiographical memory.

\section{CONCLUSIONS}

Narration is a key feature of what it means to be human [21]. While understanding the neurobiological mechanisms underlying this complex ability may seem outlandish, getting insights into the brain basis of memory or spatial exploration may indeed represent an endevour od similar complexity. Our understanding of the brain machinery enabling language processing has remarkably increased in the last two decades, largely because of methodological advances, which allow us to disegn sophisticated experiments. The time may be almost ripe to tackle the biological foundations of the "story-telling animal".

\section{REFERENCES}

1. Shi B, Cao X, Chen $Q$, et al. Different brain structures associated with artistic and scientific creativity: a voxel-based morphometry study. Sci Rep 2017;7:42911.

2. Zhu W, Chen $Q$, Xia L, et al. Common and distinct brain networks underlying verbal and visual creativity. Hum Brain Mapp 2017;38:2094-111.

3. Garrard P, Maloney LM, Hodges JR, Patterson K. The effects of very early Alzheimer's disease on the characteristics of writing by a renowned author. Brain 2004;128:250-60.

4. Price CJ. A review and synthesis of the first 20 years of PET and $f M R I$ studies of heard speech, spoken language and reading. Neuroimage 2012;62:816-47.

5. Cappa SF. Imaging semantics and syntax. Neuroimage 2012;61:427-31.

6. van Dijk TA, Kintsch W. Strategies of discourse comprehensio. New York, NY: Academic Press; 1983.
7. Zacks JM, Ferstl EC. Discourse comprehension. Neurobiology of Language: Elsevier; 2015. p. 661-73.

8. Peach RK, Coelho CA. Linking inter-and intra-sentential processes for narrative production following traumatic brain injury: Implications for a model of discourse processing. Neuropsychologia 2016;80:157-64.

9. Marini A, Spoletini I, Rubino IA, et al. The language of schizophrenia: An analysis of micro and macrolinguistic abilities and their neuropsychological correlates. Schizoph Res 2008;105:144-55.

10. Xu J, Kemeny S, Park G, et al. Language in context: emergent features of word, sentence, and narrative comprehension. Neuroimage 2005;25:1002-15.

11. Kuperberg GR, Lakshmanan BM, Caplan DN, Holcomb PJ. Making sense of discourse: An fMRI study of causal inferencing across sentences. Neuroimage 2006;33:343-61.

12. Martín-Loeches M, Casado P, Hernández-Tamames JA, ÁlvarezLinera J. Brain activation in discourse comprehension: a 3t fMRI study. Neuroimage 2008;41:614-22.

13. Smirnov D, Glerean E, Lahnakoski JM, et al. Fronto-parietal network supports context-dependent speech comprehension. Neuropsychologia 2014;63:293-303.

14. Kandylaki KD, Nagels A, Tune S, et al. Predicting "when" in discourse engages the human dorsal auditory stream: An fMRI study using naturalistic stories. J Neurosci 2016;36:12180-91.

15. Friston K, Kiebel S. Predictive coding under the free-energy principle. Philos Trans R Soc Lond B Biol Sci 2009;364:1211-21.

16. Huth AG, Nishimoto $S, V u$ AT, Gallant JL. A continuous semantic space describes the representation of thousands of object and action categories across the human brain. Neuron 2012;76:1210-24.

17. Huth AG, de Heer WA, Griffiths TL, et al. Natural speech reveals the semantic maps that tile human cerebral cortex. Nature 2016:532:453-8.

18. Hasson U, Nir Y, Levy I, et al. Intersubject synchronization of cortical activity during natural vision. Science 2004;303:1634-40.

19. Nishimoto $S, V u$ AT, Naselaris $T$, et al. Reconstructing visual experiences from brain activity evoked by natural movies. Curr Biol 2011;21:1641-6.

20. Milivojevic B, Varadinov $M$, Grabovetsky $A V$, et al. Coding of event nodes and narrative context in the hippocampus. J Neurosci 2016;36:12412-24.

21. Gottschall J. The storytelling animal: How stories make us human: Houghton Mifflin Harcourt; 2012.2016;36:12412-24. 